\title{
Sum rules for an atomic hyperfine structure in a magnetic field
}

\author{
Savely G. Karshenboim
}

\begin{abstract}
Sum rules for the energy levels of a hyperfine multiplet in a constant uniform magnetic field is presented. It is found that for any values of the electron angular moment and the nuclear spin there are certain linear combinations of energy levels which do not depend on the magnetic field and can be used to determine the unperturbated $h f s$ separation in the presence of perturbing magnetic field. It is also demonstrated that there are other linear combinations which are linear with the external magnetic field and hence can be used to determine bound values of the electron and nuclear magnetic moments. The accuracy of the approximation within which the result is valid is also discussed.
\end{abstract}

PACS Nos.: 32.10.Fn, 32.60.+i 1

Résumé : Nous ... French version of abstract (supplied by CJP)

[Traduit par la rédaction]

\section{Introduction}

An essential part of present-day high-precision frequency measurements is related to experiments performed in the presence of some residual electric or magnetic field. To reduce the uncertainty caused by the field, one tries to perform the measurement on those sublevels which are under control, e.g., for sublevels slightly affected by the perturbing field. Here, we study another possible option to deal with a residual magnetic field. We show that despite the presence of a perturbing magnetic field shifting the energy levels, there are some special combinations of energies which do not depend on the magnetic field at all. In the case of microwave measurements that may be used, e.g., to determine the hyperfine separation in the ground state. There are also some more specific combinations, and in particular ones where the dependence on the magnetic field is linear.

A constant homogenous magnetic field shifts different sublevels of hyperfine-structure $(h f s)$ multiplets differently. However, as it is well known, a specific combination of energy of the sublevels of any $n S$ state in the hydrogen atom at presence of the $d c$ homogenous magnetic field

$E_{\mathrm{hfs}}^{0}=E^{\mathbf{H}}(1,+1)+E^{\mathbf{H}}(1,-1)-E^{\mathbf{H}}(1,0)-E^{\mathbf{H}}(0,0)$

remains field-independent. Here: superscript " $\mathbf{H}$ " stands for the energy perturbed by the magnetic field $\mathbf{H}$ and " 0 " is for the unperturbed levels. We use a simplified notation in which $E^{\mathbf{H}}(1,+1)$ stands for $E^{\mathbf{H}}\left(F=1, F_{z}=+1\right)$ etc. The direction of the magnetic field is along the $z$ axis. Here $\mathbf{F}$ is the complete atomic angular moment $\mathbf{F}=\mathbf{J}+\mathbf{I}$. The electron angular moment $J$ in the case of the $S$ electron is $1 / 2$, and the nuclear magnetic moment $I$ for hydrogen is also $1 / 2$. The magnetic quantum

Received 2006. Accepted 2006.

Savely G. Karshenboim. D. I. Mendeleev Institute for Metrology, St. Petersburg 190005, Russia and Max-

Planck-Institut für Quantenoptik, Garching 85748, Germany; e-mail: sek@mpq.mpg.de 
number $F_{z}$ is a well-defined quantum number in presence of both the external magnetic field and the hyperfine interaction of the magnetic moment of the nucleus with the electron angular motion, while $F$ is not in the case when the Zeeman shifts are comparable with the hyperfine separation. Still we can mark a level with its value of $F$ at zero magnetic field. The other quantum numbers (such as the principal quantum number $n$ and orbital moment $l$ in the case of hydrogen) are not presented, but indeed it is assumed that all energies are related to the same $h f s$ multiplet.

The property of the levels in the magnetic field presented in (1) has been numerously applied, e.g., to study the hydrogen [1] and muonium [2] hyperfine structure.

Here we discuss different field-independent combinations of the energy levels for arbitrary electron states (arbitrary $J$ ) and nuclear magnetic moments $(I)$. We assume for most of our study here that any effects due to the nuclear quadrupole electric moment can be neglected and consider the magnetic field as a weak one in a sense that the field affects the levels inside the $h f s$ multiplets but does not mix different multiplets.

We also consider in this paper another kind of specific linear combinations of energy levels, which depend on a value of the magnetic field, but their dependence is linear. A ratio of two linearly-dependent combinations is indeed field-independent. Such linearly-dependent combinations can be used to determine magnetic moments of the electron shell and the nucleus. These values related to the nucleus and the electron(s) in the atom are somewhat different from their free values, being affected by binding effects. Comparing various linear combinations, one can determine a dimensionless ratio of these two magnetic moments.

Measuring various data in presence of a magnetic field, one can in principle determine a value of the field and thus to introduce corrections and arrive at unperturbed energy levels and eventually find magnetic moments. There are two main advantages of the sum rules derived in our paper.

- They are derived here for an arbitrary two-spin system without use of any perturbation techniques and hence the Zeeman shifts can be of any value compared with the hyperfine splitting.

- Because of the linearity of the sum rules the combinations can be easily calculated directly from experimental data and broadening of lines due to inhomogeneity of the field, which happens in actual experiments, could be cancelled in part.

\section{The Breit-Rabi formula}

The cancellation of the dependence on the magnetic field in (1) has been known as a specific result for the $n S$-state which originates from the Breit-Rabi formula [3, 4]

$E_{\text {magn }}^{\mathbf{H}}\left(I \pm 1 / 2, F_{z}\right)=-\mu_{\text {nucl }} H \pm \frac{\Delta E}{2} \sqrt{1+\frac{4 F_{z}}{2 I+1} x+x^{2}}$

where

$x=x(\Delta E)=\frac{\left(\mu_{J}-\mu_{\text {nucl }}\right) H}{\Delta E}$

and

$\Delta E=E^{0}(F=I+1 / 2)-E^{0}(F=I-1 / 2)$

is the $h f s$ separation of the states with $F=I \pm 1 / 2$ at zero magnetic field $\mathbf{H}$. The electron magnetic moment

$\boldsymbol{\mu}_{J}=-\mu_{J} \frac{\mathbf{J}}{J}=-g_{J} \mu_{B} \mathbf{J}$ 
depends on the electron state, mainly on values of angular momentum $J$ and orbital momentum $L$. A slight dependence on principle quantum number $n$ is to appear from perturbation theory due to relativistic and QED effects. The nuclear magnetic moment

$\boldsymbol{\mu}_{\mathrm{nucl}}=\mu_{\mathrm{nucl}} \frac{\mathbf{I}}{I}=g_{N} \mu_{N} \mathbf{I}$

also contains a weak dependence on the atomic state via the small relativistic and QED contributions to $g_{N}$. The Breit-Rabi formula is valid for a single electron atom in an $S$ state. Our approach developed further is valid for an atom with any number of electrons. Referring to 'electron' magnetic moment or 'electron' quantum numbers we will not distinguish between single-electron values and values for the electron shell.

Four energy sublevels of an $n S$ state in the hydrogen atom as a function of the magnetic field are depicted in Fig. 1. A similar behavior of six hyperfine sublevels of an $n S$ in deuterium is plotted in Fig. 2. Let us briefly discuss our notation. The energy $E^{\mathbf{H}}\left(F, F_{Z}\right)$ is not the energy of a state with a quantum numbers $F$ and $F_{z}$ because $F$ is not a well-defined quantum number in presence of the magnetic field. We consider the energy of a level as a function of the field strength $H$ and mark it with a value of $F$ at zero field when $F$ is well defined. We hope this notation is not confusing.

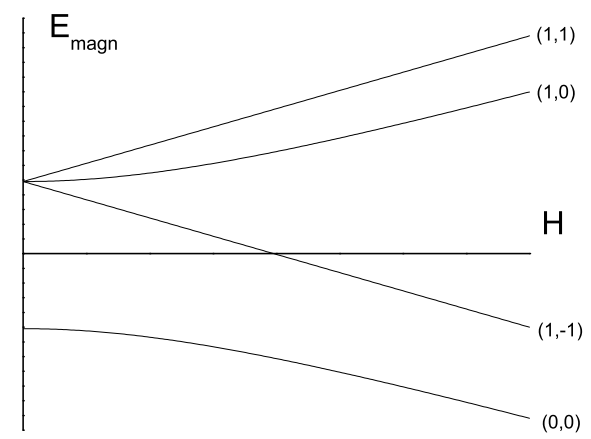

Fig. 1. Zeeman effect of the $n S$ sublevels in hydrogen (not to scale). Dependence of the energy of the ${ }^{3} S_{1}$ triplet levels and the ${ }^{1} S_{0}$ singlet level on the strength of the constant uniform field $H$ is plotted.

One can conclude from (2) that there is a specific combination of few levels for an electronic $n S$ state and any nuclear spin $I$ which is field-independent. To see that, first we note that

$E_{\text {magn }}^{\mathbf{H}}(I+1 / 2, \pm(I+1 / 2))=-\mu_{\text {nucl }} H+\frac{\Delta E}{2}(1 \pm x)$,

and thus

$E_{\text {magn }}^{\mathbf{H}}(I+1 / 2,+(I+1 / 2))+E_{\text {magn }}^{\mathbf{H}}(I+1 / 2,-(I+1 / 2))=-2 \mu_{\text {nucl }} H+\Delta E$.

Next, we find that

$E_{\text {magn }}^{\mathbf{H}}(I \pm 1 / 2,+(I-1 / 2))=-\mu_{\text {nucl }} H \pm \frac{\Delta E}{2} \sqrt{1+\frac{2(2 I-1)}{2 I+1} x+x^{2}}$,

and

$E_{\text {magn }}^{\mathbf{H}}(I+1 / 2,+(I-1 / 2))+E_{\text {magn }}^{\mathbf{H}}(I-1 / 2,(I-1 / 2))=-2 \mu_{\text {nucl }} H$. 


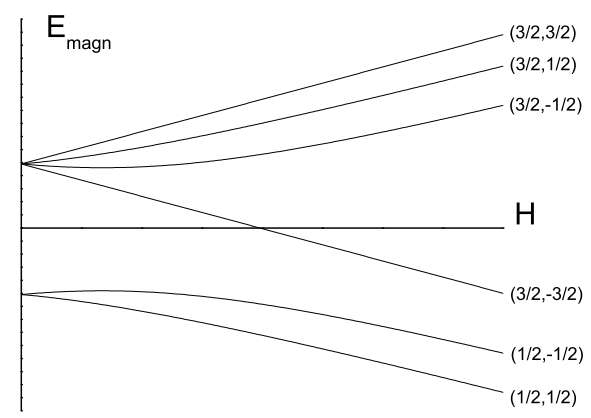

Fig. 2. Zeeman effect of the $n S$ sublevels in deuterium (not to scale).

Similarly, we obtain

$E_{\text {magn }}^{\mathbf{H}}(I+1 / 2,-(I-1 / 2))+E_{\text {magn }}^{\mathbf{H}}(I-1 / 2,-(I-1 / 2))=-2 \mu_{\text {nucl }} H$.

Finally we find two field-independent combinations:

$$
\begin{aligned}
\Delta E & =\left[E^{\mathbf{H}}(I+1 / 2,+(I+1 / 2))+E^{\mathbf{H}}(I+1 / 2,-(I+1 / 2))\right] \\
& -\left[E_{\text {magn }}^{\mathbf{H}}(I+1 / 2,+(I-1 / 2))+E_{\text {magn }}^{\mathbf{H}}(I-1 / 2,+(I-1 / 2))\right],
\end{aligned}
$$

and

$$
\begin{aligned}
\Delta E & =\left[E_{\text {magn }}^{\mathbf{H}}(I+1 / 2,+(I+1 / 2))+E_{\text {magn }}^{\mathbf{H}}(I+1 / 2,-(I+1 / 2))\right] \\
& -\left[E_{\text {magn }}^{\mathbf{H}}(I+1 / 2,-(I-1 / 2))+E_{\operatorname{magn}}^{\mathbf{H}}(I-1 / 2,-(I-1 / 2))\right],
\end{aligned}
$$

where $\Delta E$ is defined by (4).

For practical application their symmetric combination can be more appropriate

$$
\begin{aligned}
\Delta E & =\left[E_{\text {magn }}^{\mathbf{H}}(I+1 / 2,+(I+1 / 2))+E_{\text {magn }}^{\mathbf{H}}(I+1 / 2,-(I+1 / 2))\right] \\
& -\frac{1}{2}\left\{\left[E_{\text {magn }}^{\mathbf{H}}(I+1 / 2,+(I-1 / 2))+E_{\text {magn }}^{\mathbf{H}}(I+1 / 2,-(I-1 / 2))\right]\right. \\
& \left.+\left[E_{\text {magn }}^{\mathbf{H}}(I-1 / 2,+(I-1 / 2))+E_{\text {magn }}^{\mathbf{H}}(I-1 / 2,-(I-1 / 2))\right]\right\} .
\end{aligned}
$$

Recent progress in optical measurements offers an opportunity to determine $h f s$ interval of excited states studying optical transitions, like, for example, two-photon excitation $1 S \rightarrow 2 S$ in hydrogen and deuterium (see, e.g., [5]). For such a measurement the observed lines are to be related to transitions which conserve $F$ and $F_{z}$. If the measurement is performed with a residual field, the sum rules with vanishing field dependence may be helpful. It is advantageous for practical applications to express the results in terms of the absolute energy of the levels. On contrary, the energy in (2) is a relative energy defined in such a way that $E(F=I \pm 1 / 2)= \pm \Delta E$ at zero magnetic field. The complete energy is

$E\left(\lambda, F, F_{z}\right)=E_{\text {Coul }}(\lambda)+E_{\text {magn }}^{\mathbf{H}}\left(F, F_{z}\right)$, 
where $\lambda$ stands for quantum numbers which describe the energy levels neglecting hyperfine interaction and external magnetic field, i.e. $n l j$ in the case of hydrogen atom. Indeed, there is still a possibility for an arbitrary additive constant, but that should be the same for any states. Hopefully, equations (12), (13) and (14) are organized in such a way that it remains valid after a substitute

$E_{\text {magn }}^{\mathbf{H}}\left(F, F_{z}\right) \rightarrow E\left(\lambda, F, F_{z}\right)$.

\section{Atom in a magnetic field: an arbitrary electronic state}

The field-independent results in (12) and (14) have been obtained from an explicit expression for the energy levels (2) [3]. Now we are to show that such a kind of expressions can be derived in a more general case for an arbitrary electronic state and any value of the nuclear spin. The only limitation is a complete neglection of the quadrupole contribution (if it is present), and thus the separations between levels at zero magnetic field are determined by a single parameter, which is the nuclear magnetic moment. We consider an atom in a constant uniform magnetic field which induces the energy shifts much smaller than the fine structure, so we can study a reduced Hamiltonian defined as an operator in a certain spin and angular-momentum space for a particular electron state

$H_{\text {magn }}=-\left(\boldsymbol{\mu}_{J} \cdot \mathbf{H}\right)-\left(\boldsymbol{\mu}_{\text {nucl }} \cdot \mathbf{H}\right)+(\mathbf{J} \cdot \mathbf{I}) A$,

where $A$ is the $h f s$ constant which depends on the electron state. $H_{\text {magn }}$ is an operator in the space of the spin and angular-momentum degrees of freedom. There are two natural basic sets to describe all such states. One, $\left(F, F_{z}\right)$, is appropriate for low magnetic field, while the other, $\left(I_{z}, J_{z}\right)$, is a proper one in the case when the Zeeman effect dominates over the $h f s$ separations. An important point is that $F_{z}$ is a good quantum number for both sets and

$\left[F_{z}, H_{\text {magn }}\right]=0$.

We demonstrate now, that even in the case of arbitrary values of the nuclear spin and the electron angular moment, a derivation similar to that for (12) is still possible. It may be constructed in a similar way with four (six) energy levels. To show that we remind, that, since $F_{z}$ is a good quantum number, it is unnecessary to diagonalize the Hamiltonian $H_{\text {magn }}$ for all $h f s$ states simultaneously. One can do that sector by sector, with each sector being related to a particular value of $F_{z}$. There is no interference between sectors different values of $F_{z}$.

Let us return to the previously discussed well-known result for an $n S$ state (see (12) and (14)). Technically, it is has been successfully derived because of two basic properties of Breit-Rabi expression (2) for energy levels at magnetic field.

- There is only a single state with $F_{z}=+(I+1 / 2)$ and its energy has a linear dependence on magnetic field. The same is for the state with $F_{z}=-(I+1 / 2)$. The field-dependence of the energy is presented in (7). Since, no re-diagonalization has been involved the dependence remains linear.

- There are two states for each other values of $F_{z}$ and in particular for $F_{z}=+(I-1 / 2)$. To diagonalize the related Hamiltonian one needs to deal with $2 \times 2$ matrices only. In a basis of $\left(F, F_{z}\right)$ states, the diagonal matrix elements are related to the hyperfine interaction, while the off-diagonal elements are results of interaction with the magnetic field. The same is true for the substates with $F_{z}=-(I-1 / 2)$. The result of re-diagonalization is given by (9).

Returning to an arbitrary state, we note that there is a certain similarity. The $F$ value varies between $F_{\max }=I+J$ and $F_{\min }=|I-J|$ with step $\Delta F=1$. The maximal value of $F_{z}\left(F_{z}=+(I+J)\right)$ is 
possible only for $F=F_{\max }$. The same is correct for $F_{z}=-(I+J)$. Since for these values there is a single hyperfine sublevel for each, the energy dependence is trivial (cf. (7))

$E_{\text {magn }}^{\mathbf{H}}(I+J, \pm(I+J))=-\mu_{\text {nucl }} H+\frac{\Delta E_{1}}{2}\left(1 \pm x_{1}\right)$,

Similarly to the states $F_{z}= \pm(I+1 / 2)$ in the previous consideration, the field dependence is linear. There are two parameters introduced here: $\Delta E_{1}=2 E_{\text {magn }}^{0}(F=I+J)$ is the energy for the levels with maximal angular momentum $F=F_{\max }$ at zero field, and $x_{1}=x\left(\Delta E_{1}\right)$.

The energy $E_{\text {magn }}^{0}$ can be in principle defined in an arbitrary way and as matter of fact it actually cancels out in $\Delta E_{1} x_{1}$ term in (19), while any additive constant has sense only if we discuss a few levels, not a single one. We postpone any exact definition of $E_{\text {magn }}^{0}$ until it will be necessary for further consideration.

Considering $F_{z}=F_{\max }-1$, we note that there are now two states with $F=F_{\max }$ and $F=$ $F_{\max }-1$, which are split at zero magnetic field. The same situation is indeed for $F_{z}=-\left|F_{\max }-1\right|$ That is similar to $F_{z}= \pm(I-1 / 2)$. The energy levels are (cf. (9))

$E_{\text {magn }}^{\mathbf{H}}(I+J-1 / 2 \pm 1 / 2,(I+J-1))=\bar{E}-\mu_{\text {nucl }} H \pm \frac{\Delta E_{2}}{2} \sqrt{1+C(I, J) \cdot x_{2}+x_{2}^{2}}$.

Here $\Delta E_{2}=E_{\text {magn }}^{0}(F=I+J)-E_{\text {magn }}^{0}(F=I+J-1)$ is the $h f s$ splitting between levels with $F=F_{\max }$ and $F=F_{\max }-1$;

$\bar{E}=\frac{E_{\text {magn }}^{0}(F=I+J)+E_{\text {magn }}^{0}(F=I+J-1)}{2} ;$

and we still do not need to specify $E_{\mathrm{magn}}^{0}$. In other words, it is not important for this part of consideration from what level we count the energy.

We do not specify also constant $C(I, J)$ in the equation above, which depends on $I$ and $J$, since that is not necessary for our further evaluations.

Limiting our consideration by these six states (with $F_{z}= \pm F_{\max }$ and $F_{z}= \pm\left|F_{\max }-1\right|$, we easily derive expressions similar to (12), (13) and (14).

Firstly, we note that

$\Delta E_{1} \cdot x_{1}=\Delta E_{2} \cdot x_{2}$.

Secondly, we choose such a definition of the energy for a moment, that satisfies the condition

$\bar{E}=0$,

which is a way to fix an additive constant in definition of energy. Once we do so, the equations (19) and (20) take now the form (cf. (7) and (8))

$$
\begin{aligned}
E_{\text {magn }}^{\mathbf{H}}(I+J, \pm(I+J)) & =-\mu_{\text {nucl }} H+\frac{\Delta E_{2}}{2}\left(1 \pm x_{2}\right), \\
E_{\text {magn }}^{\mathbf{H}}(I+J-1 / 2 \pm 1 / 2,(I+J-1)) & =-\mu_{\text {nucl }} H \pm \frac{\Delta E_{2}}{2} \sqrt{1+C(I, J) \cdot x_{2}+x_{2}^{2}},
\end{aligned}
$$

and the result

$$
\begin{aligned}
\Delta E_{2} & =\left[E_{\text {magn }}^{\mathbf{H}}(I+J,+(I+J))+E_{\text {magn }}^{\mathbf{H}}(I+J,-(I+J))\right] \\
& -\frac{1}{2}\left\{\left[E_{\text {magn }}^{\mathbf{H}}(I+J,+(J+I-1))+E_{\text {magn }}^{\mathbf{H}}(F=I+J,-(I+J-1))\right]\right. \\
& \left.+\left[E_{\text {magn }}^{\mathbf{H}}(I+J-1,+(I+J-1))+E_{\text {magn }}^{\mathbf{H}}(I+J-1,-(I+J-1))\right]\right\}
\end{aligned}
$$


becomes obvious.

Above we have defined the energy by (21); however, we note that (22) (cf. (14)) allows a redefinition of energy by introducing an additive constant. That reads that we are free to change definition of energy with an additive constant if necessary and this constant can depend on any quantum numbers not involved into hyperfine interaction.

\section{Sum rules: field-independent combinations}

Above we have taken advantage of similarity in certain expressions for simple previously known case and for a general situation. The similarity is related to sectors determined by a value of $F_{z}$ which have one or two substates only. That is the case of two maximal possible values of $F_{z}$ (and the opposite values with negative $F_{z}$ ). Any other sector with a lower value of $\left|F_{z}\right|$ involves more than two states and any explicit expression for energy becomes more complicated. It is even unclear whether it is possible to obtain such expressions. Below we develop an alternative approach to derive various field-independent values as linear combinations of energies in a constant uniform magnetic field. The approach does not need any explicit expressions for energy levels at magnetic field.

First, we find a trace of the magnetic Hamiltonian

$S_{0}=\operatorname{Sp}\left\{H_{\text {magn }}\right\}=\sum_{\zeta}\left\langle\zeta\left|H_{\text {magn }}\right| \zeta\right\rangle$

where the summation is performed over a complete basis set of sublevels, $\zeta$, of the $h f s$ multiplet. There are two basis sets useful for calculations: one is related to states with a fixed values of $\mathbf{F}^{2}$ and $F_{z}$ and the other is for the states with determined values of $J_{z}$ and $I_{z}$. Since the summation is over a complete set the result does not depend on the choice between these two sets.

It is obviously that

$S_{0}=\operatorname{Sp}\left\{H_{\text {magn }}\right\}=0$.

A more general trace of interest is

$S_{2 n}=\operatorname{Sp}\left\{F_{z}^{2 n} H_{\text {magn }}\right\}$.

Still, the field-dependent part obviously vanishes because

$\operatorname{Sp}\left\{F_{z}^{2 n} \boldsymbol{\mu}_{J} \cdot \mathbf{H}\right\}=\operatorname{Sp}\left\{F_{z}^{2 n} \boldsymbol{\mu}_{\text {nucl }} \cdot \mathbf{H}\right\}=0$.

The remaining part is field-independent

$S_{2 n}=\operatorname{Sp}\left\{F_{z}^{2 n}(\mathbf{J} \cdot \mathbf{I})\right\} A=\frac{1}{2} \sum_{F, F_{z}}\left\{F_{z}^{2 n}[F(F+1)-I(I+1)-J(J+1)]\right\} A$.

The $h f s$ constant $A$ determines all energy separations inside the $h f s$ multiplet and e.g. one can find

$E_{\text {magn }}^{0}(F=I+J)-E_{\text {magn }}^{0}(F=|I-J|)=\frac{1}{2}[(I+J)(I+J+1)-(|I-J|)(|I-J|+1)] A$

Let us discuss values which can be measured directly. The complete Hamiltonian is of the form

$H_{\text {tot }}=H_{0}+H_{\text {magn }}$,

where the first term depends on the electronic state and does not depend on magnetic parameters $\mathbf{I}$ and $\mathbf{J}$, while the second term depends on them only, but not on detail of the atomic state. One can see that

$\operatorname{Sp}\left\{F_{z}^{2 n}\left(H_{0}+H_{\text {magn }}\right)-\overline{F_{z}^{2 n}}\left(H_{0}+H_{\text {magn }}\right)\right\}=S_{2 n}+H_{0} \operatorname{Sp}\left(F_{z}^{2 n}-\overline{F_{z}^{2 n}}\right)-\overline{F_{z}^{2}} S_{0}=S_{2 n}$ 
where $\overline{F_{z}^{2 n}}$ is an average value over all the states

$\overline{F_{z}^{2 n}}=\frac{\operatorname{Sp}\left\{F_{z}^{2 n}\right\}}{(2 J+1)(2 I+1)}$.

After some transformations we arrive to a final field-independent sum rule

$$
\begin{aligned}
S_{2 n}=\sum_{F, F_{z}}\left(F_{z}^{2}-\overline{F_{z}^{2 n}}\right) E^{\mathbf{H}}\left(F, F_{z}\right) & =\frac{1}{2} \sum_{F, F_{z}}\left\{F_{z}^{2 n}(F(F+1)-I(I+1)-J(J+1))\right\} \\
& \times \frac{E_{\operatorname{magn}}^{0}(F=I+J)-E_{\operatorname{magn}}^{0}(|I-J|)}{(I+J+1)(I+J)-(|I-J|+1)|I-J|},
\end{aligned}
$$

where the sum is over all spin states. The equation for $S_{2}$ in the case of hydrogen reproduces (1) and in the case of deuterium (14).

\section{Sum rules: combinations linear in magnetic field}

The relation (32), valid for an arbitrary value of $n$, allows to obtain an infinite number of relationships. Obviously, not all of them are independent ${ }^{1}$ of each other. Let us discuss a number of independent identities. The equation, presented above, combines the values

$\widetilde{E}_{\text {magn }}^{\mathbf{H}}\left(F_{z}\right)=\sum_{F}\left\{E_{\text {magn }}^{\mathbf{H}}\left(F,+F_{z}\right)+E_{\text {magn }}^{\mathbf{H}}\left(F,-F_{z}\right)\right\}$

for not-negative $F_{z}$. The number of independent identities, derived in such a way, cannot exceed the number of different values of $\widetilde{E}^{\mathbf{H}}\left(F_{z}\right)$. The latter is equal to $I+J+1$ for integer $I+J$ and $I+J+1 / 2$ for semi-integer. We note that all identities allow free additive normalization of energy. It means that we, e.g., should consider $\widetilde{E}_{\text {magn }}^{\mathbf{H}}\left(F_{z}\right)-\widetilde{E}_{\text {magn }}^{\mathbf{H}}(0)$ rather than $\widetilde{E}_{\text {magn }}^{\mathbf{H}}\left(F_{z}\right)$, which reduces number of independent sum rules by unity.

Comparing with consideration for $n S$ states, we note that the field-independent combination is not necessarily a symmetrical sum of the contributions with $F_{z}$ and $-F_{z}$ (cf. (12) and (13)). Meantime, we derived above only symmetric combinations. Asymmetric field-independent combinations cannot be found this way.

Nevertheless, we can study odd sum rules related to the properties of

$S_{2 n+1}=\operatorname{Sp}\left\{F_{z}^{2 n+1} H_{\text {magn }}\right\}$.

In contrast to the even sum rules (25), the part related to the hyperfine interaction of the angular momentum and the nuclear magnetic moment vanishes

$\operatorname{Sp}\left\{F_{z}^{2 n}(\mathbf{J} \cdot \mathbf{I})\right\} A=0$,

and the rest depends on the magnetic field. The dependence is quite simple:

$S_{2 n+1}=\sum_{J_{z}, I_{z}}\left(J_{z}+I_{z}\right)^{2 n+1}\left\{-g_{j} \mu_{B} J_{z}+g_{N} \mu_{N} I_{z}\right\} H$.

It is linear in the magnetic field.

1 We understand independent relations as relations which present independent linear combinations of $E_{\text {magn }}^{\mathbf{H}}\left(F, F_{z}\right)$. Indeed, they are not independent in a sense that all are eventually expressed in terms of the hyperfine constant $A$. 
In such a way we have derived not more than $I+J$ values for integer $I+J$ and $I+J+1 / 2$ values of $S_{2 n+1}$ for semi-integer $I+J$, which are linear in magnetic field. Any ratio of them is indeed a field-independent quantity. That provides us with another set of values, which cannot be affected by the magnetic field. So, we derived not more than $I+J-1$ and $I+J-1 / 2$ field-independent values independent from each other.

We note that

$$
\sum_{J_{z}, I_{z}}\left(J_{z}+I_{z}\right)^{2 n+1}=0
$$

and thus

$S_{2 n+1}=\sum_{J_{z}, I_{z}}\left(J_{z}+I_{z}\right)^{2 n+1} E_{\mathrm{magn}}^{\mathbf{H}}\left(J_{z}, I_{z}\right)$,

where we changed the notation and $E^{\mathbf{H}}\left(J_{z}, I_{z}\right)$ stands for the energy of the levels which for the strong magnetic field is characterized by eigenvalues of $J_{z}$ and $I_{z}$.

\section{Summary}

In our evaluation above we marked the levels with quantum numbers $\left(F, F_{z}\right)$ or $\left(I_{z}, J_{z}\right)$, which both are not good at the presence at the same time of two effects such as the hyperfine interaction and interaction with the magnetic field. The indeces $F, I_{z}$ and $J_{z}$ corresponded not to well-defined quantum numbers, but were just indexes related to some limits ('weak' or 'strong' magnetic field). In the case of any formal problem of derivation of our results for $S_{2 n}$ and $S_{2 n+1}$ we note that all of them can be presented as a double sum, when the internal sum is a sum of all states, $\zeta\left(F_{z}\right)$, with a fixed value of $F_{z}$

$\bar{E}\left(F_{z}\right)=\sum_{\zeta\left(F_{z}\right)} E_{\text {magn }}^{\mathbf{H}}\left(\zeta\left(F_{z}\right)\right)$,

while the external sum is over $F_{z}$ which is a good quantum number. In such a way we need only somehow to identify levels and know their eigennumbers for $F_{z}$.

Altogether we derived not more than $2(I+J)-1$ field-independent values (as $S_{2 n}$ or as ratio of two $S_{2 n+1}$ ). The sum rules, derived here, (for $I \geq 1$ and $J \geq 1$ ) are not covered by the Breit-Rabi formula. We also suggested an analog of the Breit-Rabi formula for six specific levels. The derivation above is valid for any spin hamiltonian when we can neglect interaction between different multiplets.

In conclusion, let us discuss two problems related to possible corrections to the sum rules due to

- effects of the nuclear structure;

- quantum electrodynamics effects and atomic structure.

We note that the nuclear electric quadrupole moment (or higher order electric and magnetic moments) affects the identities above but still the field-dependent combinations exist. Since $F_{z}$ is a good quantum number, the sums $S_{2 n}$ are field-independent and calculating all of them one can find contribution of all moments separately. For instance, the nuclear quadrupole moment contributes to hyperfine structure if $I \geq 1$ and $J \geq 1$. The magnetic Hamiltonian becomes of the form

$H_{\text {magn }}=-\left(\boldsymbol{\mu}_{J} \cdot \mathbf{H}\right)-\left(\boldsymbol{\mu}_{\mathrm{nucl}} \cdot \mathbf{H}\right)+(\mathbf{J} \cdot \mathbf{I}) A+4(\mathbf{J} \cdot \mathbf{I})^{2} B$

where the constant $B$ is directly related to the quadrupole contribution to the hyperfine structure. In such a case, the sums $S_{2 n}$ remains field-independent, but they are expressed linearly in terms of $A$ and $B$ (cf. (27)). It is enough to find two field-independent values, e.g., $S_{2}$ and $S_{4}$, to determine two 
contributions to the hyperfine structure separately: one caused by the dipole magnetic moment and the other caused by the electric quadrupole moment, i.e., by $A$ and $B$. We do not present any explicit relationships but it is clear that they can be presented if it would be really necessary for any particular applications. The sum rules offer an accurate method to compare the magnetic dipole moment and electric quadrupole moment in the presence of a magnetic field. It can be also used to compare magnetic moments of the nucleus and an electron by studying a ratio of linear combinations. If one measures magnetic field, the linear combinations can be helpful as well (cf., e.g., with a high-precision determination of the muonium magnetic moment in Ref [2]).

There is only a source of corrections to the derived sum rules due to other levels. If the magnetic shift of levels of fine and gross structure is much smaller than the distance between those levels, we can separate the levels in theoretical consideration to study their magnetic structure. If the shift is big, a number of levels are mixed (cf. the Paschen-Back effect) and our evaluation is incorrect. It also leads to a Hamiltonian non-linear in the magnetic field. Indeed, in a low field such effects produce certain corrections to the Hamiltonian (17). Additionally, these effects produce a shift of the center of gravity of magnetic multiplet. However, as long as these effects are small they can be taken into account perturbatively as effects in the second order in the magnetic field.

All other effects, such as effects of quantum electrodynamics, can only affect the values of the electron and nuclear magnetic moments which become slightly different from the free values and thus introduce some corrections to the energy levels in the magnetic field. However, the structure of the Hamiltonian (17) is the same and sum rules for the field-independent contributions are not affected.

\section{Acknowledgments}

The work was supported in part by RFBR (under grant \# 06-02-16156) and by DFG (under grant \# DFG GZ: 436 RUS 113/769/0). I am grateful to Gordon Drake and Vladimir Ivanov for useful discussions.

\section{References}

1. P. F. Winkler, D. Kleppner, T. Myint and F. G. Walther, Phys. Rev. A 5, 83 (1972).

2. V. W. Hughes and G. zu Putlitz, In Quantum Electrodynamics, ed. by T. Kinoshita (World Scientific, Sinpagore, 1990) p. 822.

3. G. Breit and I. Rabi, Phys. rev. 38, 2082 (1931).

4. H. A. Bethe and E. E. Salpeter: Quantum Mechanics of One- and Two-electon Atoms (Plenum, NY, 1977).

5. N. Kolachevsky, M. Fischer, S. G. Karshenboim and T. W. Hänsch, Phys. Rev. Lett. 92, 033003 (2004); N. Kolachevsky, P. Fendel, S. G. Karshenboim and T. W. Hänsch, Phys. Rev. A 70, 062503 (2004). 